\title{
Sub-Centimeter Spatial Resolution in Distributed Fiber Sensing Based on Dynamic Brillouin Grating in Optical Fibers
}

\author{
Sanghoon Chin, Nikolay Primerov, and Luc Thévenaz
}

\begin{abstract}
Optical fiber sensors based on stimulated Brillouin scattering in optical fibers have now clearly demonstrated their excellent capability for long-range distributed strain and temperature measurements. The fiber is used as sensing element and a value for temperature and/or strain can be obtained from any point along the fiber. While the spatial resolution of classical configurations is practically limited to 1 meter by the phonon lifetime, novel approaches have been demonstrated these past years that can overcome this limit. In this paper, this could be achieved by two physical processes: prior activation of a steady acoustic wave through the classical Brillouin interaction between two Brillouin pumps, and interrogation by Bragg reflection on the acoustic wave using a distinct ultra-short pulse in a highly birefringent fiber. We could achieve a spatial resolution below one centimeter, while preserving the full accuracy on the determination of temperature and strain.
\end{abstract}

Index Terms-Distributed fiber sensor, fiber optics, nonlinear optics, optical fiber sensor, stimulated Brillouin scattering.

\section{INTRODUCTION}

D ISTRIBUTED fiber sensing based on stimulated Brillouin scattering in optical fibers was first proposed in the late $1980 \mathrm{~s}$ as an alternative technique to the classical optical time domain reflectometer (OTDR) to measure local attenuation along an optical fiber [1]. It turned out rapidly that it had many more potentialities for sensing, since Brillouin scattering is intrinsically very sensitive to temperature and the deformations experienced by the fiber [2], [3]. This comes from the property that Brillouin scattering must satisfy a very strict phase matching condition, making the interaction observable as a resonance spectrally spreading over a very narrow band (typically $27 \mathrm{MHz}$ at a wavelength of $1550 \mathrm{~nm}$ ). For this reason, many different configurations based on stimulated Brillouin scattering were proposed to improve sensing performance in terms of measurement range, spatial resolution and measurement time [4]-[8]. However, in most time-domain Brillouin distributed sensors, it remains still a scientific challenge to

\footnotetext{
Manuscript received November 18, 2010; revised February 22, 2011; accepted February 26, 2011. Date of publication March 10, 2011; date of current version December 01, 2011. This work was supported by Omnisens and the partners in the COST Action 299 "FiDES." The associate editor coordinating the review of this paper and approving it for publication was Prof Jose Lopez Higuera.

The authors are with the EPFL Swiss Federal Institute of Technology, Institute of Electrical Engineering, 1015 Lausanne, Switzerland (e-mail: sanghoon. chin@epfl.ch).

Color versions of one or more of the figures in this paper are available online at http://ieeexplore.ieee.org.

Digital Object Identifier 10.1109/JSEN.2011.2126568
}

overcome the spatial resolution limitation resulting from the finite phonon lifetime, which is approximately $1 \mathrm{~m}$ (equivalent to a 10-ns-long pump pulse) observed as an excess spectral broadening of the Brillouin resonance.

An alternative approach based on dynamic Brillouin grating distributed sensing (DBG-DS) has been recently proposed using a highly birefringent fiber and has experimentally demonstrated temperature and/or strain measurements with a high spatial resolution, and even distributed birefringence measurements in polarization maintaining fibers [9]-[13]. The spatial resolution using this type of sensing mechanism was soon identified as physically limited only by the motion of the acoustic wave that is much lesser than a millimeter. In this paper, the results are obtained using the same principle of distributed fiber sensing as presented in former papers [9], [13], but by consolidating with more details the physical principles behind dynamic Brillouin grating sensing. In addition, various sensing illustrative applications are presented, taking advantage of a 5.5-mm extreme spatial resolution, which is the best ever reported to our knowledge in any time-domain Brillouin distributed sensor.

\section{PRINCIPLE OF DBG-DS}

Stimulated Brillouin scattering in optical fibers is an efficient nonlinear interaction between two counter-propagating optical waves (a strong pump at frequency $\nu_{\text {pump }}$ and a weak probe at frequency $\nu_{\text {probe }}$ ), mediated by an acoustic wave. Once a specific phase matching condition is met, given by the proper matching of the optical and acoustic wavelengths, themselves related to the waves' frequencies by their specific velocities, a periodic acoustic density wave appears along the fiber due to the electrostriction process. It is only realized for a strict spectral separation between the two optical waves $\left(\Delta \nu=\nu_{\text {pump }}-\right.$ $\nu_{\text {probe }}$ ) that must be equal to the acoustic wave frequency $\nu_{B}=$ $2 \nu_{\text {pump }} V_{a} / c\left(\nu_{B}\right.$ is called the Brillouin frequency shift and $V_{a}$ is the acoustic velocity) [14], [15]. In turn this density wave results in a modulation of the refractive index that acts as a moving grating reflector realizing the exact Bragg condition for the two optical waves. Since the Bragg reflector is moving at the acoustic velocity the scattered light experiences a Doppler shift, so that eventually a perfect in-phase coupling is achieved from the higher frequency wave to the lower frequency phase, and a perfect $\pi$-phase coherent superposition for the reverse coupling. The net result of the interaction is the lower frequency optical wave experiences an exponential growth, designated as 

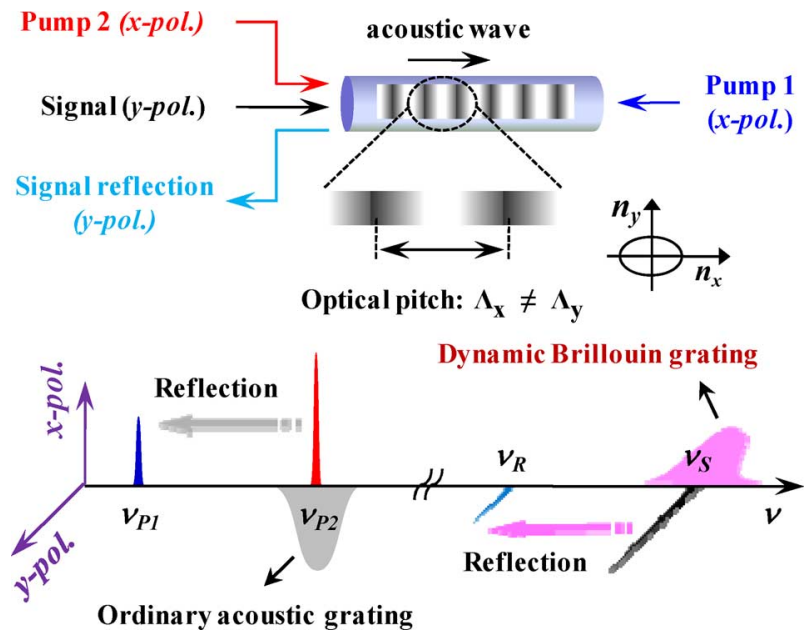

Fig. 1. Principle to generate a dynamic Brillouin grating (DBG) in a polarization maintaining fiber, based on the ordinary process of stimulated Brillouin scattering, and to realize a reflection of an orthogonally polarized signal wave. $\nu_{B}=\nu_{P 1}-\nu_{P 2}=\nu_{S}-\nu_{R}\left(\nu_{R}\right.$ being the light frequency of the signal reflection), where $\nu_{B}$ is Brillouin frequency shift of the fiber.

SBS amplification. On the contrary, the higher frequency optical wave experiences an exponential attenuation, so that SBS can also be assimilated to an attenuation process, which is designated as SBS loss. The pump can be chosen to be the higher or the lower frequency wave, the response is perfectly symmetric on the probe and of identical amplitude in a low gain configuration.

Now let us consider the particular case when an acoustic grating is generated through the SBS process in a highly birefringent fiber such as a polarization maintaining fiber (PMF). Fig. 1 describes the configuration of optical frequencies and states of polarization for two pumps and signal waves. Two counter- propagating pump waves (Pump 1 at $\nu_{P 1}$ and probe at $\nu_{P 2}$, and $\left.\nu_{B}=\nu_{P 2}-\nu_{P 1}\right)$ interact to create the acoustic grating while the two pumps propagate along one primary polarization axis of the PMF. Since the role of these two interacting waves is to essentially generate an acoustic wave of the largest possible amplitude, requiring preferably their powers to be of similar magnitude, they are both designated as "pumps." In such a situation, a light signal traveling along the orthogonal polarization axis with respect to the pumps is of great interest, since its light can be reflected by the grating. However, it must be noticed that the optical frequency of this signal light $\nu_{S}$ has to be different to the orthogonally polarized pumping waves, since the Bragg condition is satisfied at a distinct frequency as a result of the difference in the refractive index between the slow and fast polarization axes [16]. Actually, the properties of the grating in terms of spectral bandwidth and reflectance can be dynamically controlled by simply varying the time waveform of the two pump waves, thereby designated as dynamic Brillouin grating (DBG).

The central frequency of the dynamic grating can be simply calculated by the expression [16]

$$
\Delta \nu=\nu_{S}-\nu_{P 1}=\frac{\Delta n}{n_{g}} \nu_{P 1}
$$
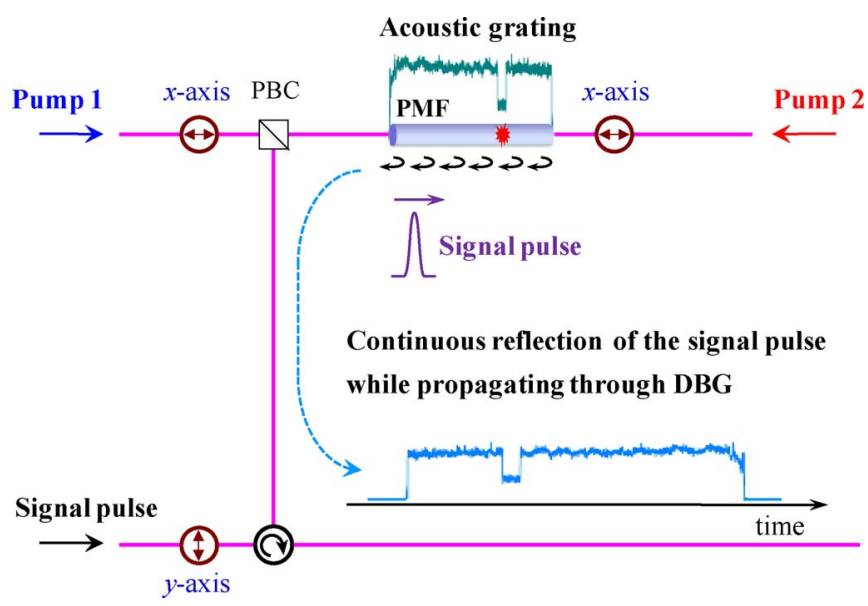

Fig. 2. Schematic illustration of a DBG-based distributed sensor, where the two $\mathrm{CW}$ pumps and signal pulse are aligned along $x$-axis and $y$-axis, respectively. PBS: polarization beam combiner. PMF: polarization maintaining fiber.

where $n_{g}$ is the group index. This expression basically results from the strict frequency matching condition for stimulated Brillouin scattering in optical fibers [14], [15]

$$
\nu_{B}=\frac{2 V_{a}}{c} n_{x} \nu_{P 1}^{x}=\frac{2 V_{a}}{c} n_{y} \nu_{S}^{y} .
$$

$n_{x}$ and $n_{y}$ being the refractive indices along the slow and fast axes of the fiber, respectively, the superscripts ${ }^{x, y}$ representing the polarization direction of the optical waves and $V_{a}$ being the acoustic velocity. It can be clearly seen in (1) that the signal must be placed at an up-shifted frequency above the pump due to the simple fact that $n_{x}>n_{y}$. On the other hand, it is obvious that when the polarization states of the pumps and signal are swapped, i.e., the grating is generated by two pumps that are $y$-polarized, the signal frequency must be down-shifted below the pump frequency without changing the spectral distance between Pump 1 and the signal given by (1).

This type of sensing technique presents crucial advantages by two aspects due to the fact that the physical processes of the DBG creation and its interrogation are absolutely distinct. First, the spectral bandwidth of the Brillouin grating is uniquely determined by its optical length, itself fixed by the duration of the pumps [17], [18]. In general, continuous waves (CW) can be utilized as Brillouin pumps, but a pulsed wave is preferable for Pump 2, co-propagating and just anticipating the signal pulse, to avoid any possible depletion and an excessive gain for Pump 1. Nonetheless this pulse shows a duration far exceeding the acoustic life time ( $>10 \mathrm{~ns}$ ) to allow a full building of the acoustic wave. Therefore, the generated DBG is continuously present along the entire fiber as far as the signal pulse is concerned, while keeping a narrow Brillouin gain spectrum. Second, the spatial resolution in this measurement is essentially determined by the duration of the signal pulse. It must be pointed out that the signal pulse is only used to probe the local grating amplitude, so its duration can be shortened to any desired value, leading to the possibility to achieve a considerably shorter spatial resolution while maintaining a good accuracy on the determination of the Brillouin shift $\nu_{B}$. Actually, the DBG acts as a Bragg reflector and its spectral transfer function can be fully 


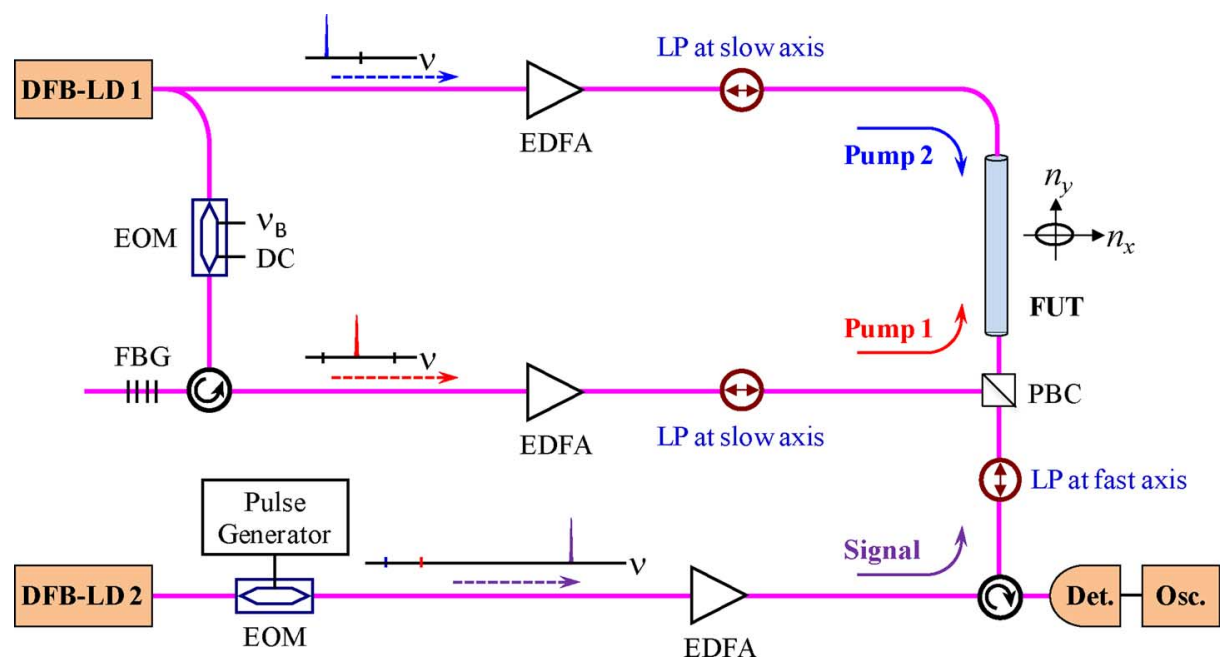

Fig. 3. Block diagram of the dynamic Brillouin grating-based distributed sensor using a polarization maintaining fiber as sensing element. EOM: electro-optic modulator. FBG: fiber Bragg grating. EDFA: erbium-doped fiber amplifier. LP: linear polarizer. FUT: fiber under test. PBC: polarization beam combiner.

compared to a weak fiber Bragg grating written in an optical fiber [17]. So, it can be assumed that the signal pulse propagates through a very long weak Bragg grating, and the signal pulse is continuously reflected by the short fraction of the grating covered by the pulse. It is possible to obtain this way a high spatial resolution map of the local Brillouin grating amplitude along the fiber. In summary, the grating is generated by quasi-CW waves and its local amplitude will only depend on the Brillouin gain spectrum at the given position. The very short signal pulse experiences a reflection proportional the local grating amplitude and its backscattered time distribution directly provides a map of the acoustic wave local amplitude. By then changing the frequency difference between Pumps 1 and 2, it is possible to activate acoustic waves with different frequencies and retrieve the local Brillouin gain spectrum that shows a strong dependence on temperature and strain.

The principle of operation of a DBG-based distributed sensor, as described above, is graphically illustrated in Fig. 2, where the red spot represents an externally applied temperature or strain. The SBS interaction between two CW pumps creates a DBG all along the fiber with a constant reflectivity if the Brillouin frequency shift of the fiber does not vary along the fiber. So, the signal pulse will be continuously back-reflected while propagating through the grating. However, the pulse reflection substantially drops in presence of a hot spot, since the Brillouin frequency shift in this section is different and so the Brillouin gain that determines the strength of the grating vanishes. Therefore, by simply mapping the time trace to the fiber distance, one can find the spatial position of a particular heating event in the fiber. On the other hand, the time trace can be inversed so as to show a maximum reflection in the heating section when the frequency difference between two pumps is set at the Brillouin frequency in this section. All Brillouin frequency configurations can be addressed by scanning the frequency difference between the 2 pumps and the local Brillouin spectrum can be thus conveniently retrieved. From the spectrum the central frequency is determined that gives a measure of temperature or strain.

\section{EXPERIMENTAL LAYOUT AND RESULTS}

The experimental layout of the DBG-based distributed sensor we implemented is depicted in Fig. 3. A commercial distributedfeedback laser diode (DFB-LD) at $1551 \mathrm{~nm}$ is used as a light source. The higher branch is boosted using an erbium-doped fiber amplifier (EDFA) to generate Brillouin Pump 1. In addition, Pump 1 is sent into a linear polarizer to align the state of polarization along the $x$-axis. Then Pump 1 is delivered into one end of the PMF. The optical power of Pump 1 before entering the fiber is controlled by a variable optical attenuator and is set to $20 \mathrm{dBm}$. The lower branch is externally modulated through an electro-optic modulator (EOM) around the range of possible Brillouin frequencies $v_{B}$ and a DC bias is adequately applied to the modulator, resulting in a complete suppression of the optical carrier. So, at the output of the EOM, only two sidebands are present at frequencies $\pm v_{B}$ below and above the optical carrier frequency, respectively. Then the higher frequency sideband is precisely selected using a tunable fiber Bragg grating. In turn, the optical power is also amplified by another EDFA, generating Brillouin Pump 2. This way the two Brillouin pumps can be exactly separated by frequency differences close to the Brillouin frequency shift, and also their spectral distance remains intrinsically highly stable [5]. The state of polarization of Pump 2 is also aligned along the $x$-axis in the same manner, before entering into the fiber from the other end of the fiber at a similar 20-dBm power level.

The signal pulse is then generated using the gain switching technique by simply modulating the injection current applied to another DFB laser diode with closely matching wavelength. This way a signal pulse train with a 55-ps FWHM duration is generated, as shown in Fig. 4, at a repetition rate of $25 \mathrm{MHz}$ (40-ns period), which is much longer than the transit time of $2.1 \mathrm{~ns}$ through the entire sensing fiber. So, it is secured that only one signal pulse propagates through the entire sensing fiber. The central frequency of the signal pulse is precisely controlled by the temperature applied to the laser diode, for a perfect centering of the signal frequency on the middle of the grating resonance. 


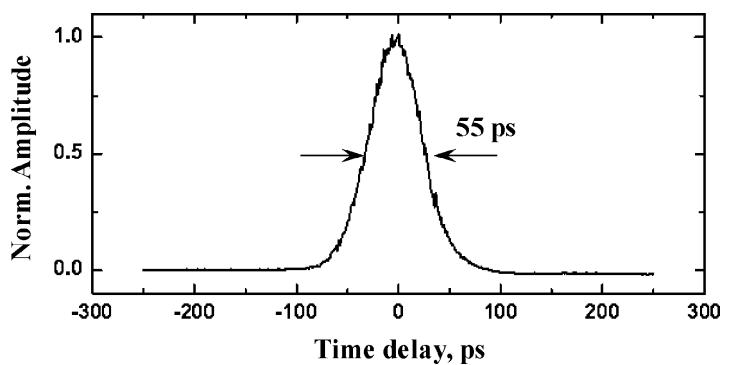

Fig. 4. Temporal power distribution of the signal pulse showing a FWHM duration of $55 \mathrm{ps}$, generated by gain switching technique. Such a pulse gives a spatial resolution of the measurement of $5.5 \mathrm{~mm}$.

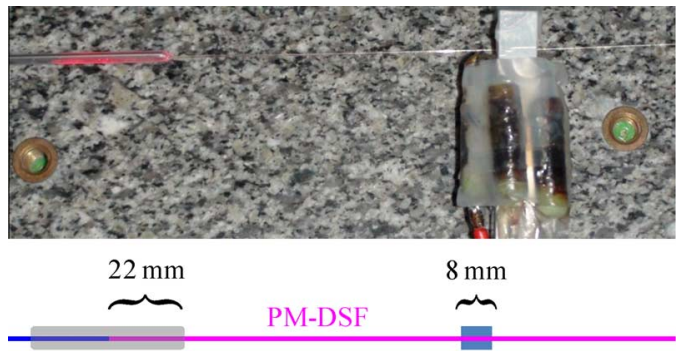

Splicing point

Hot spot

Fig. 5. Image showing the implementation of a localized hot spot, placed at the beginning of the sensing fiber. An 8 -mm-long fiber section was temperaturecontrolled to present a localized sharp temperature transition along the sensing fiber.

It was found that the DBG is spectrally positioned at around $45 \mathrm{GHz}$ above the Pump 1 frequency, determined by the fiber birefringence. Then the signal pulse is strongly amplified by a high gain EDFA, resulting in a peak power of about $20 \mathrm{~W}$ necessary to counterbalance the weak reflectivity of the grating. The state of polarization of the signal pulse is controlled to be orthogonally aligned with respect to the two pumps, hence linearly polarized along the $y$-axis. The signal pulse is then combined with Pump 1 using a polarization beam combiner (PBC) and launched into the PM-DSF to interrogate the distributed acoustic grating amplitude with a spatial resolution of $5.5 \mathrm{~mm}$.

A 42-cm-long polarization maintaining dispersion shifted fiber (PM-DSF) is used in this experiment as a sensing fiber (fiber under test, FUT) and the Brillouin characteristics of the fiber were measured, showing a Brillouin frequency shift $v_{B}$ of $10.56 \mathrm{GHz}$ and a Brillouin gain FWHM bandwidth of $30 \mathrm{MHz}$. The layout of the FUT is shown in Fig. 5. The PM-DSF is spliced to a different type of PMF, showing a distinct Brillouin shift and the splicing is protected using a plastic heat shrink tube. Then, we create a very local hot spot over $8 \mathrm{~mm}$ of fiber using heating resistors at a $77-\mathrm{mm}$ distance from the splicing. Moreover, the sensing fiber is in direct tight contact to a bulk stone optical table to avoid the typical heat propagation from the heated segment to the rest of the fiber, which is normally observed as a smooth transition of the Brillouin frequency shift in the vicinity of the heating position.

To realize distributed measurements along the fiber, the temporal trace of the reflection while the signal pulse propagates through the DBG is acquired using a fast detector with 32 times averaging and a sampling rate of $40 \mathrm{GSa} / \mathrm{s}$, equivalent to $\sim$ 4 points $/ \mathrm{cm}$. Actually, a temporal trace is acquired for a set

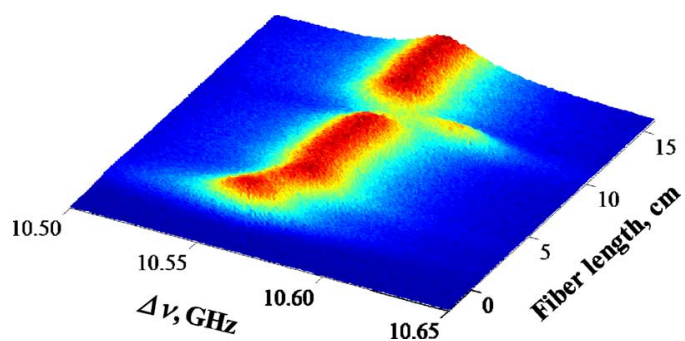

Fig. 6. 3-D map of the Brillouin gain spectrum along the initial part of the sensing fiber containing the hot spot, obtained with 32 times averages.

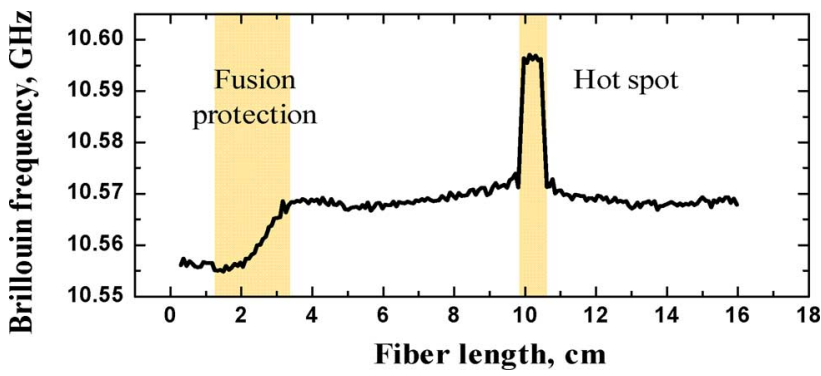

Fig. 7. Distributed measurement of the Brillouin frequency shift along the sensing fiber by the system based on dynamic Brillouin grating, showing a sharp transition of the Brillouin frequency at the hot spot position. The compressive strain effect of the splice protection sleeve can also be observed.

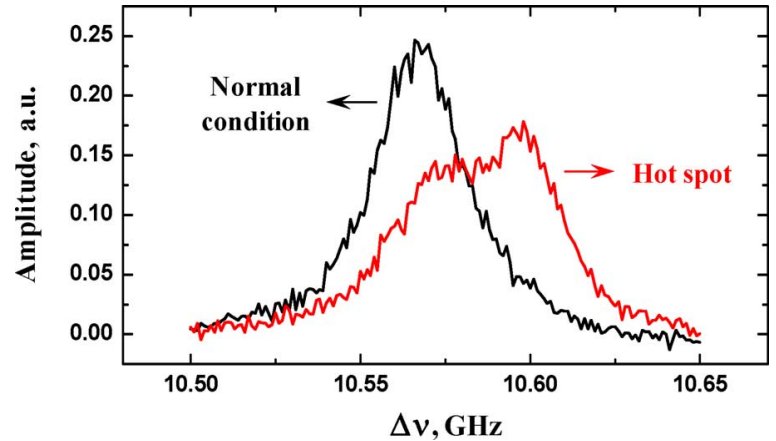

Fig. 8. Measured local BGSs at the position of the hot spot (in red) and a position at ambient temperature (in black).

of frequency spacings $\Delta \nu$ between Pump 1 and Pump 2 by incrementing $\Delta \nu$ from 10.50 to $10.65 \mathrm{GHz}$ by $1-\mathrm{MHz}$ steps. Fig. 6 shows a 3-D map of the distributed Brillouin gain spectrum (BGS) along the initial part of the sensing fiber, approximately the section shown in Fig. 5. It is immediately visible that the BGS near the 8-mm heated section is clearly separated from the rest of the fiber that remains under normal environmental condition, hence verifying the high spatial resolution of this system. The distribution of exact Brillouin frequency shift was calculated from the measurement as a function of position with a spatial resolution of $5.5 \mathrm{~mm}$, as shown in Fig. 7. One can see a sharp step transition of Brillouin frequency appearing at the heating position, which apparently results from the temperature variation. The amount of increment in Brillouin frequency shift was measured to be $+28 \mathrm{MHz}$, which corresponds to a temperature change of $+30 \mathrm{~K}$ according to results of previous measurements [9].

The local BGSs at two different positions under an ambient temperature and a heating section are shown in Fig. 8. A clear shift between the peak frequencies of the two BGSs is observed 


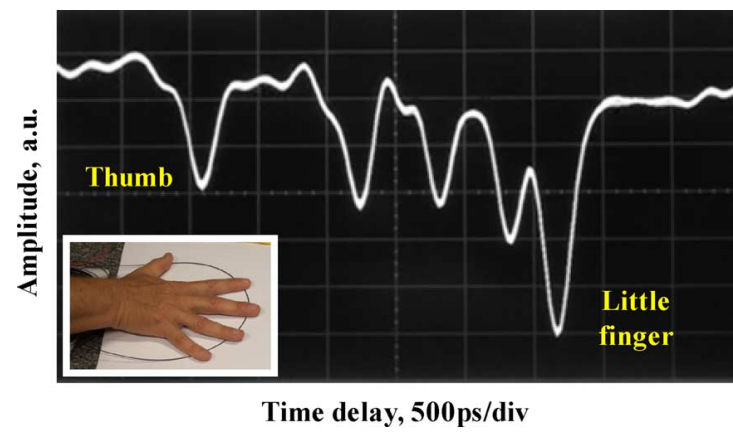

Fig. 9. Temporal distribution of the signal pulse reflection along the sensing fiber when five fingers from one hand are placed on the fiber, as shown in the inset image. The pump frequency difference is adjusted to give a maximal reflection at ambient temperature. The local heating of the fingers is sufficient to create enough detuning which results in a localized drop of the Brillouin grating reflectivity exactly at the fingers position.

without a significant broadening of the spectrum in the hot spot, showing a spectral width of $52 \mathrm{MHz}$. In a standard time-domain Brillouin sensor, an equivalent spatial resolution using the same signal pulse would be associated with a FWHM bandwidth of 8-GHz spectrum, showing that the accuracy on the Brillouin frequency determination is obviously maintained together with the achievement of a high spatial resolution. It must be noticed that the spectral profile of the BGS reflects the temperature distribution within the spatial resolution and, despite our efforts, clearly a non-uniform temperature profile is realized in the hot spot as evidenced by the distorted Brillouin gain spectrum. It remains very challenging to make a uniform and sharp thermal transition in an optical fiber over a few $\mathrm{mm}$, since at that scale heat conduction is turning important and the diffusion length is calculated to be about $3 \mathrm{~mm}$ in a silica fiber of standard size. It must be pointed out that the central frequency of the DBG shifts in an inversely proportional manner to the temperature change, due to the dependence of the fiber birefringence on external temperature, showing a typical value of $50 \mathrm{MHz} / \mathrm{K}$ [19]. Actually, the decrease of the peak amplitude observed in our experiment can be simply explained by this overall shift of dynamic grating spectrum. But the very broad 8-GHz spectral width of the signal pulse secures a sufficient reflection over a fairly large temperature range.

It is also interesting to observe that a linear variation of the Brillouin frequency is also observed in Fig. 8 within a $10-\mathrm{mm}$ long region located at the splice protection section, resulting in $1 \mathrm{MHz} / \mathrm{mm}$. This gradual decrease of the Brillouin frequency probably results from a compressive strain, possibly resulting from the cooling of the heat shrunk protection. In a previous study, the Brillouin frequency dependence on strain was experimentally evaluated, resulting in the value of $0.05 \mathrm{MHz} / \mu \varepsilon$ [5], [20]. So, we can estimate that the residual stress induced by the heat shrunk protection might be of the order of $200 \mu \varepsilon$.

As an illustration of the effective sub-centimetric spatial resolution and the strict localization of the dynamic Brillouin gratings a randomly spaced successive temperature change along the sensing fiber has been tested. The spatial sequence of temperature steps was realized by simply touching the PMF with the five fingers, so that the contacting points are heated by the normal human body temperature, as shown in the inset of Fig. 9. The frequency difference between the two pumps $\Delta \nu$ was set to lead to a maximum reflection at ambient temperature. So, the signal pulse experienced less reflection while propagating under the fingers. This results in five dips in the time trace shown in Fig. 9, which represent the localized heating events, so equivalent to the individual positions of the five fingers. The relative spacing of the fingers can be easily checked by the positioning of the dips.

\section{CONCLUSION}

We have experimentally demonstrated for the first time using a time-domain coding sub-centimetric distributed Brillouin fiber sensing, using a sensing system based on dynamic Brillouin grating in polarization maintaining fibers. In this type of distributed sensing system, the duration of the signal pulse has no impact on the measurement accuracy, since this pulse is not involved in the generation of the acoustic grating, hence the spectral bandwidth of the measured Brillouin resonance is not modified by the power spectrum of the signal pulse. It means that the spatial resolution of this system can be ultimately improved, possibly down to a sub-millimeter scale. However, some effects turn non-negligible at such small scale, such as the thermal diffusion, the strain profile through the fiber section and the acoustic wave propagation during the phonon lifetime $(\sim 0.1 \mathrm{~mm})$. By reaching this spatial resolution we think to be close of the limit where the fiber dimensions start to be non-negligible and thus to impact on the measurement.

\section{REFERENCES}

[1] T. Horiguchi and M. Tateda, "Optical-fiber-attenuation investigation using stimulated Brillouin scattering between a pulse and a continuous wave," Opt. Lett., vol. 14, pp. 408-410, 1989.

[2] T. Horiguchi, T. Kurashima, and M. Tateda, "A technique to measure distributed strain in optical fibers," IEEE Photon. Technol. Lett., vol. 2, no. 5, pp. 352-354, May 1990.

[3] T. Kurashima, T. Horiguchi, and M. Tateda, "Distributed-temperature sensing using stimulated Brillouin scattering in optical silica fibers,' Opt. Lett., vol. 15, pp. 1038-1040, 1990.

[4] X. Bao, D. J. Webb, and D. A. Jackson, "22-km distributed temperature sensor using Brillouin gain in an optical fiber," Opt. Lett., vol. 18, pp. 552-554, 1993

[5] M. Nikles, L. Thevenaz, and P. A. Robert, "Brillouin gain spectrum characterization in single mode optical fibers," J. Lightw. Technol., vol. 15, no. 10, pp. 1842-1851, Oct. 1997.

[6] K. Hotate and T. Hasegawa, "Measurement of Brillouin gain spectrum distribution along an optical fiber using a correlation-based technique - Proposal, experiment and simulation," IEICE Trans. Electron., vol. E83-C, pp. 405-412, 2000.

[7] M. N. Alahbabi, Y. T. Cho, and T. P. Newson, "150-km-range distributed temperature sensor based on coherent detection of spontaneous Brillouin backscatter and in-line Raman amplification," J. Opt. Soc. Amer. B, vol. 22, pp. 1321-1324, 2005.

[8] K. Y. Song, Z. He, and K. Hotate, "Distributed strain measurement with millimeter-order spatial resolution based on Brillouin correlation domain analysis," Opt. Lett., vol. 31, pp. 2526-2528, 2006.

[9] K. Y. Song, S. Chin, N. Primerov, and L. Thévenaz, "Time-domain distributed fiber sensor with $1 \mathrm{~cm}$ spatial resolution, based on Brillouin dynamic gratings," J. Lightw. Technol., vol. 28, no. 14, pp. 2062-2067, July15 2010.

[10] W. Zou, Z. He, and K. Hotate, "Complete discrimination of strain and temperature using Brillouin frequency shift and birefringence in a polarization maintaining fiber," Opt. Exp., vol. 17, pp. 1248-1255, 2009.

[11] Y. Dong, X. Bao, and L. Chen, "Distributed temperature sensing based on birefringence effect on transient Brillouin grating in a polarizationmaintaining photonic crystal fiber," Opt. Lett., vol. 34, pp. 2590-2592, 2009.

[12] Y. Dong, X. Bao, and L. Chen, "Truly distributed birefringence measurement of polarization-maintaining fibers based on transient Brillouin grating," Opt. Exp., vol. 35, pp. 193-195, 2010.

[13] K. Y. Song and H. J. Yoon, "High-resolution Brillouin optical time domain analysis based on Brillouin dynamic grating," Opt. Lett., vol. 35, pp. 52-54, 2010. 
[14] R. W. Boyd, Nonlinear Optics, 2nd ed. New York: Academic, 2003.

[15] G. P. Agrawal, Nonlinear Fiber Optics, 2nd ed. San Diego, CA: Academic, 1995.

[16] K. Y. Song, W.Zou, Z. He, and K. Hotate, “All-optical dynamic grating generation based on Brillouin scattering in polarization maintaining fiber," Opt. Lett., vol. 33, pp. 926-928, 2008.

[17] K. Y. Song and H. J. Yoon, "Observation of narrowband intrinsic spectra of Brillouin dynamic gratings," Opt. Lett., vol. 17, pp. 2958-2960, 2010.

[18] Y. Dong, L. Chen, and X. Bao, "Characterization of the Brillouin grating spectra in a polarization-maintaining fiber," Opt. Exp., vol. 18, pp. 18960-18967, 2010.

[19] W. Zou, Z. He, K. Y. Song, and K. Hotate, "Correlation-based distributed measurement of a dynamic grating spectrum generated in stimulated Brillouin scattering in a polarization-maintaining optical fiber," Opt. Lett., vol. 34, pp. 1126-1128, 2009.

[20] K. Y. Song, K. Lee, and S. B. Lee, "Tunable optical time delays based on Brillouin dynamic grating in optical fibers," Opt. Exp., vol. 17, pp. 10344-10349, 2009.

Sanghoon Chin received the M.S. degree in information and communication from Gwangju Institute of Science and Technology (GIST), Gwanju, Korea, in 2005 and the Ph.D. degree in electrical engineering department from Ecole Polytechnique Fédérale de Lausanne (EPFL), Lausanne, Switzerland, where he developed his expertise on slow and fast light. His research was mainly oriented to progress the dynamic control of speed of a light signal in optical fibers, using stimulated Brillouin scattering in fibers.

Currently, he is a Postdoctoral Researcher at EPFL. He is implementing Brillouin slow light into microwave photonics for RF signal processing such as photonic microwave filters and phased array antennas. In 2010, He made several academic visits to the Polytechnique University in Valencia, Valencia, Spain, and at Thales Research and Technology, Palaiseau, France, respectively, and improved his expertise on broadband microwave signal processing based on photonic delay line.
Nikolay Primerov received the M.S. degree in chemical technology of monocrystals for electronics from Ural Federal University, Yekaterinburg, Russia, in 2005. He is currently pursuing the Ph.D. degree at Swiss Federal Institute of Technology of Lausanne (EPFL), Lausanne, Switzerland.

Since 2008, he has been working as a Ph.D. assistant in the Group for Fiber Optics, EPFL. His current research is concentrated on investigation of dynamic Brillouin grating in optical fibers and its applications for photonic delay lines, optical signal processing, and distributed fiber sensing.

Luc Thévenaz received the M.Sc. degree in 1982 and the Ph.D. degree in physics in 1988, both from the University of Geneva, Geneva, Switzerland.

In 1988, he joined the Swiss Federal Institute of Technology of Lausanne (EPFL), Lausanne, Switzerland, where he currently leads a research group involved in photonics, namely fiber optics and optical sensing. Research topics include Brillouin-scattering fiber sensors, slow and fast light, nonlinear fiber optics, and laser spectroscopy in gases. He achieved with his collaborators the first experimental demonstration of optically controlled slow and fast light in optical fibers, and is at the origin of innovative configurations for Brillouin distributed fiber sensors, such as generation of the signal wave using modulation sidebands and Brillouin dynamic gratings. During his career he stayed at Stanford University, Korea Advanced Institute of Science and Technology (KAIST), Tel Aviv University, and the University of Sydney. In 2000, he cofounded the company Omnisens that is developing and commercializing advanced photonic instrumentation.

Prof. Thévenaz is Chairman of the European COST Action 299 "FIDES: Optical Fibers for New Challenges Facing the Information Society" and author or coauthor of some 300 publications and five patents. He is Fellow of the Optical Society of America. 